\title{
Comparison of SUPG with Bubble Stabilization Parameters and the Standard SUPG
}

\author{
Xiaowei Liu ${ }^{1}$ and Jin Zhang ${ }^{2}$ \\ ${ }^{1}$ Department of Mathematics, Tongji University, Shanghai 200092, China \\ ${ }^{2}$ School of Mathematical Sciences, Shandong Normal University, Jinan 250014, China \\ Correspondence should be addressed to Xiaowei Liu; xwliuvivi@hotmail.com
}

Received 23 February 2014; Accepted 13 May 2014; Published 2 June 2014

Academic Editor: Gerassimos Barbatis

Copyright (c) 2014 X. Liu and J. Zhang. This is an open access article distributed under the Creative Commons Attribution License, which permits unrestricted use, distribution, and reproduction in any medium, provided the original work is properly cited.

We study a streamline upwind Petrov-Galerkin method (SUPG) with bubble stabilization coefficients on quasiuniform triangular meshes. The new algorithm is a consistent Petrov-Galerkin method and shows similar numerical performances as the standard SUPG when the mesh Péclet number is greater than 1. Relationship between the new algorithm and the standard SUPG will be explored. Numerical experiments support these results.

\section{Introduction}

We consider the reaction-convection-diffusion problem in $2 \mathrm{D}$

$$
\begin{aligned}
-\varepsilon \Delta u+\mathbf{b} \cdot \nabla u+c u & =f, \quad(x, y) \in \Omega, \\
u & =0, \quad(x, y) \in \partial \Omega,
\end{aligned}
$$

where $\Omega$ is a bounded open domain in $\mathbb{R}^{2}$, with the boundary $\partial \Omega, \mathbf{b} \in\left(W_{1}^{\infty}(\Omega)\right)^{2}, c \in L^{\infty}(\Omega)$ are given functions, and $\varepsilon \ll|\mathbf{b}|$ is a small positive parameter. For simplicity, we only consider the case that $\Omega=(0,1)^{2}$. In the following, suppose that there is a constant $\mu_{0}>0$ such that

$$
c-\frac{1}{2} \nabla \cdot \mathbf{b} \geq \mu_{0}>0, \quad \forall(x, y) \in \Omega .
$$

It is also assumed that $f$ is sufficiently smooth. In the case of $\mathbf{b}=\left(b_{1}, b_{2}\right)^{T}>(0,0)^{T}\left(b_{1}, b_{2} \geq \beta\right.$, where $\beta$ is a positive constant), the solution of (1) typically has two exponential layers of width $O(\varepsilon \ln (1 / \varepsilon))$ at the sides $x=1$ and $y=$ 1 of $\Omega$. In the case of $\mathbf{b}=(b, 0)^{T}(b \geq \beta>0)$, the solution of (1) typically has an exponential layer of width $O(\varepsilon)$ near the outflow boundary at $x=1$ and two characteristic (or parabolic) layers of width $O(\sqrt{\varepsilon})$ near the characteristic boundaries at $y=0$ and $y=1$.
When the mesh Péclet number Pe is greater than 1 , there exist global unphysical oscillations in numerical solutions of standard discretization schemes on general meshes. Hence, stabilized methods and/or a priori adapted meshes are widely used in order to get discrete solutions with satisfactory accuracy. An overview on these methods can be found in the survey $[1,2]$.

One of the most famous stabilized finite element methods is the streamline upwind Petrov-Galerkin method (SUPG). The SUPG proposed by Hughes and Brooks [3] is known to provide good stability properties and high accuracy. However, there are several drawbacks in SUPG, such as lacking discrete maximum principle and involving second derivatives and difficulties in determining the stabilization coefficients. Driven by these problems, many researchers were devoted to improving the SUPG. A lot of numerical methods were proposed, such as SOLD [4], nonlinear residual [5], and LPS [6]. Also, relations of SUPG and other numerical formulation, like residual-free bubble method [7] and variational multiscale method [8], were studied to seek possible directions of improvement.

By means of residual, the SUPG adds to the original bilinear form a term which introduces a suitable amount of artificial viscosity in the direction of streamlines. Also, the SUPG can be viewed as an inconsistent Petrov-Galerkin 
method, since its modified weighting function cannot apply to the diffusive term (see the details in Section 4).

For this reason, we are to analyze the SUPG with bubble stabilization coefficients in 2D and compare its numerical performance with the SUPG's. From theoretical analysis and numerical results, we find that the new scheme is classified into the consistent Petrov-Galerkin formulation (CPGF) and behaves as well as SUPG. Also, the standard finite element method (FEM), which shows excellent performances for $\mathrm{Pe} \leq$ 1 , can be classified into the CPGF and viewed as a special "SUPG with bubble stabilization coefficients" by taking $\delta_{K}=$ 0 in (7). Thus, the FEM and our new scheme could be viewed as two reference numerical methods in the CPGF. This provides possibilities of constructing new numerical schemes between them in the CPGF. In fact, in our forthcoming works, we obtain a linear maximum-principle-preserving stabilized method in the CPGF by means of the FEM and the SUPG with bubble stabilization coefficients, which shows better numerical performances than the standard SUPG. Thus, our results in this paper can be viewed as a starting point to construct new numerical schemes in the CPGF.

The remainder of this paper is organized as follows. In Section 2 we formulate the problem and introduce notation and mesh. Theoretical results including stability analysis and energy norm estimates can be found in Section 3. Section 4 is devoted to the relationship between SUPG, standard finite element method, and our method. Finally, numerical experiments that illustrate our theoretical results are presented in Section 5 .

\section{Mesh and Numerical Formulation}

First we define a finite element space on triangular meshes

$$
V_{h}:=\left\{v_{h} \in C(\bar{\Omega}):\left.v_{h}\right|_{\partial \Omega}=0,\left.v_{h}\right|_{K} \text { is linear, } \forall K \in \mathscr{T}_{h}\right\},
$$

where the term "linear" is to be understood in the usual isoparametric sense. Here we assume that the triangulations $\mathscr{T}_{h}$ on $\Omega$ are quasiuniform:

$$
h_{K}>\alpha_{0} h, \quad \alpha_{K}>\alpha_{0},
$$

for any $K \in \mathscr{T}_{h}$, where $\alpha_{0}$ is a positive constant and $h_{K}, \alpha_{K}, h$ denote, respectively, the diameter of $K$, the smallest angle of $K$, and the maximum of all diameters of triangles in $\mathscr{T}_{h}$.

Using the linear finite element space $V_{h}$, we can state the standard Galerkin discretisation of (1) which reads as follows.

Find $u_{h} \in V_{h}$ such that for all $v_{h} \in V_{h}$,

$$
a_{h}\left(u_{h}, v_{h}\right)=f_{h}\left(v_{h}\right),
$$

where $a(u, v)=\varepsilon(\nabla u, \nabla v)+(\mathbf{b} \cdot \nabla u+c u, v)$.

The SUPG consists in adding to the original bilinear form a term which introduces a suitable amount of artificial viscosity in the direction of streamlines. In this case, the SUPG reads as follows.

Find $u_{h} \in V_{h}$ such that for all $v_{h} \in V_{h}$,

$$
a_{h}\left(u_{h}, v_{h}\right)=f_{h}\left(v_{h}\right) \text {, }
$$

where

$$
\begin{gathered}
a_{h}\left(u_{h}, v_{h}\right)=a\left(u_{h}, v_{h}\right) \\
+\sum_{K}\left(-\varepsilon \Delta u_{h}+\mathbf{b} \cdot \nabla u_{h}+c u_{h}, \delta_{K} \mathbf{b} \cdot \nabla v_{h}\right)_{K}, \\
f_{h}\left(v_{h}\right)=\left(f, v_{h}\right)+\sum_{K}\left(f, \delta_{K} \mathbf{b} \cdot \nabla v_{h}\right)_{K} .
\end{gathered}
$$

In (7) the term $\delta_{K}$ is defined as $\delta_{K}(x, y):=\delta_{K}=$ $C_{K}^{*} h \lambda_{1} \lambda_{2} \lambda_{3},(x, y) \in K$ in which $C_{K}^{*}$ is a constant to be determined and $\lambda_{i}(i=1,2,3)$ are the linear basis functions. Actually, $\delta_{K}$ is a bubble function. Moreover,

$$
\lambda_{1} \lambda_{2} \lambda_{3} \leq \frac{1}{27} \quad \text { in } K, \quad \int_{K} \lambda_{1} \lambda_{2} \lambda_{3}=\frac{S(K)}{60}
$$

where $S(K)$ represents the area of $K$.

Finally, we define a special energy norm (SD norm) associated with $a_{h}(\cdot, \cdot)$ :

$$
\left\|\left|u_{h}\left\|^{2}:=\varepsilon\left|u_{h}\right|_{1}^{2}+\sum_{K}\right\| \delta_{K}^{1 / 2} \mathbf{b} \cdot \nabla u_{h}\left\|_{K}^{2}+\right\| u_{h} \|^{2}\right.\right.
$$

We denote by $\|\cdot\|_{D}$ the $L^{2}$ norm in $L^{2}(D)$; that is,

$$
\|v\|_{D}^{2}=(v, v)_{D} \quad \forall v \in L^{2}(D) .
$$

If $D=\Omega$, then we drop $\Omega$ from the notation.

\section{Stability and Energy Estimates}

Throughout this subsection, we assume $C$ is some positive constant.

3.1. Stability Analysis. The stability properties are a consequence of the following.

Lemma 1. Let the parameter $C_{K}^{*}$ in $\delta_{K}$ satisfies

$$
C_{K}^{*} \leq \frac{27 \mu_{0}}{h C_{K}^{2}}
$$

for each $K \in \mathscr{T}_{h}$, where $C_{K}:=\|c\|_{L^{\infty}, K}$. Then the discrete bilinear form is coercive; that is,

$$
a_{h}\left(v_{h}, v_{h}\right) \geq \frac{A}{2}\left\|v_{h}\right\|^{2}
$$

where $A:=\max \left\{\mu_{0}, 1\right\}$.

Proof. By divergence theorem we obtain

$$
\begin{aligned}
a_{h}\left(v_{h}, v_{h}\right)= & \varepsilon\left|v_{h}\right|_{1}^{2}+\int_{\Omega}\left(c-\frac{1}{2} \nabla \cdot \mathbf{b}\right) v_{h}^{2} \\
& +\sum_{K}\left\|\delta_{K}^{1 / 2} \mathbf{b} \cdot \nabla v_{h}\right\|_{K}^{2}+\sum_{K} \int_{K} c v_{h} \delta_{K} \mathbf{b} \cdot \nabla v_{h} .
\end{aligned}
$$


Obviously the sum of first three terms is greater than $A\|\| v_{h}\|\|^{2}$ from the condition of (2), and we only need to estimate the last term. Using Hölder inequality, we have

$$
\begin{aligned}
& \left|\sum_{K} \int_{K} c v_{h} \delta_{K} \mathbf{b} \cdot \nabla v_{h}\right| \\
& \quad \leq \sum_{K}\left(\int_{K} c^{2} v_{h}^{2} \delta_{K}\right)^{1 / 2}\left(\int_{K}\left(\mathbf{b} \cdot \nabla v_{h}\right)^{2} \delta_{K}\right)^{1 / 2} \\
& \quad \leq \frac{1}{2} \sum_{K}\left[\int_{K} c^{2} v_{h}^{2} \delta_{K}+\int_{K}\left(\mathbf{b} \cdot \nabla v_{h}\right)^{2} \delta_{K}\right] \\
& \leq \frac{\mu_{0}}{2}\left\|v_{h}\right\|^{2}+\frac{1}{2} \sum_{K}\left\|\delta_{K}^{1 / 2} \mathbf{b} \cdot \nabla v_{h}\right\|_{K}^{2} \\
& \leq \frac{A}{2}\left\|v_{h}\right\|^{2},
\end{aligned}
$$

where (14) is based on the definition of $\delta_{K},(8)$, and (11):

$$
\begin{aligned}
\sum_{K} \int_{K} c^{2} v_{h}^{2} \delta_{K} & \leq C_{K}^{2} \sum_{K} \int_{K} C_{K}^{*} h \lambda_{1} \lambda_{2} \lambda_{3} v_{h}^{2} \\
& \leq \sum_{K} \int_{K} \mu_{0} v_{h}^{2}=\mu_{0}\left\|v_{h}\right\|^{2} .
\end{aligned}
$$

Then the proof of the lemma is finished.

3.2. Energy Norm Estimate. We denote by $u^{I}$ the nodal piecewise linear interpolant to $u$ over $\mathscr{T}_{h}$. From Lemma 1 and the fact of $a_{h}\left(u-u_{h}, u^{I}-u_{h}\right)=0$ one gets

$$
\begin{aligned}
\frac{\mu_{0}}{2}\|\| u^{I}-u_{h} \|\left.\right|^{2} & \leq a_{h}\left(u^{I}-u_{h}, u^{I}-u_{h}\right) \\
& =a_{h}\left(u^{I}-u, u^{I}-u_{h}\right) .
\end{aligned}
$$

Denote $\eta:=u^{I}-u, e:=u^{I}-u_{h}$ and estimate the right-hand side of (16) term by term:

$$
\varepsilon(\nabla \eta, \nabla e) \leq \varepsilon^{1 / 2}|\eta|_{1}\||e|\|,
$$

$$
\begin{aligned}
(\mathbf{b} & \cdot \nabla \eta+c \eta, e) \\
& =\int_{\partial \Omega} \mathbf{b} \cdot \mathbf{n} \eta e-\int_{\Omega} \nabla \cdot \mathbf{b} \eta e-\int_{\Omega}(\mathbf{b} \cdot \nabla e) \eta+\int_{\Omega} c \eta e \\
& =\int_{\Omega}(c-\nabla \cdot \mathbf{b}) \eta e-\int_{\Omega}(\mathbf{b} \cdot \nabla e) \eta \\
& \leq C\left(\int_{\Omega} \eta^{2}\right)^{1 / 2}\left(\int_{\Omega} e^{2}\right)^{1 / 2}+\left(\int_{\Omega}(\mathbf{b} \cdot \nabla e)^{2}\right)^{1 / 2}\left(\int_{\Omega} \eta^{2}\right)^{1 / 2} \\
& \leq C h^{2}\||| e\|\left|+C h^{-1 / 2}\|\| e\left\|h^{2} \leq C h^{3 / 2}\right\|\right| \mid e\|\|,
\end{aligned}
$$

where we have used the standard interpolation results $\|\eta\| \leq$ $\mathrm{Ch}^{2}$ (see [9]) and the first inequality of (18) is obtained by

$$
\begin{aligned}
\int_{\Omega} & (\mathbf{b} \cdot \nabla e)^{2} \leq C \beta \int_{\Omega}(\nabla e)^{2} \\
& =C h^{-1} \beta(\nabla e)^{2} \sum_{K} \int_{K} \delta_{K} \\
& \leq C h^{-1} \sum_{K} \int_{K} \delta_{K}(\mathbf{b} \cdot \nabla e)^{2} \leq C h^{-1}\|e\|^{2} \\
\sum_{K} & \left(\varepsilon \Delta u+\mathbf{b} \cdot \nabla \eta+c \eta, \delta_{K} \mathbf{b} \cdot \nabla e\right) \\
& \leq \sum_{K} \int_{K}(C \varepsilon+\mathbf{b} \cdot \nabla \eta+c \eta) \delta_{K}^{1 / 2} \delta_{K}^{1 / 2} \mathbf{b} \cdot \nabla e \\
& \leq \sum_{K}\left\|(C \varepsilon+\mathbf{b} \cdot \nabla \eta+c \eta) \delta_{K}^{1 / 2}\right\|_{K}\left\|\delta_{K}^{1 / 2} \mathbf{b} \cdot \nabla e\right\|_{K} \\
& \leq\left(\sum_{K}\left\|\delta_{K}^{1 / 2}(C \varepsilon+\mathbf{b} \cdot \nabla \eta+c \eta)\right\|_{K}^{2}\right)^{1 / 2}\left(\sum_{K}\left\|\delta_{K}^{1 / 2} \mathbf{b} \cdot \nabla e\right\|_{K}^{2}\right)^{1 / 2} \\
& \leq C h^{2}\|e\| \|
\end{aligned}
$$

Combining all of these estimates, one gets

$$
\left\|u-u_{h}\right\| \mid \leq C h^{3 / 2}
$$

\section{Comparison of SUPG with Bubble Stabilization Coefficients and the Standard SUPG}

Consider the bilinear form of SUPG:

$$
\begin{aligned}
& \varepsilon\left(\nabla u_{h}, \nabla v_{h}\right)+\left(\mathbf{b} \cdot \nabla u_{h}+c u_{h}, v_{h}\right) \\
& \quad+\sum_{K} \delta_{K}\left(-\varepsilon \Delta u_{h}+\mathbf{b} \cdot \nabla u_{h}+c u_{h}, \mathbf{b} \cdot \nabla v_{h}\right)_{K} \\
& =\left(f, v_{h}\right)+\sum_{K}\left(f, \delta_{K} \mathbf{b} \cdot \nabla v_{h}\right)_{K},
\end{aligned}
$$

where $\delta_{K}$ is constant in $K$.

It can be rewritten in the form

$$
\begin{aligned}
& \varepsilon\left(\nabla u_{h}, \nabla v_{h}\right)+\left(\mathbf{b} \cdot \nabla u_{h}+c u_{h}, v_{h}+\tilde{\delta} \mathbf{b} \cdot \nabla v_{h}\right) \\
& \quad=\left(f, v_{h}+\widetilde{\delta} \mathbf{b} \cdot \nabla v_{h}\right)
\end{aligned}
$$

where $\left.\widetilde{\delta}\right|_{K}=\delta_{K}$. 
Notice that (22) does not correspond to a consistent Petrov-Galerkin formulation in general except in the case when $\mathbf{b} \equiv \mathbf{C}$ and $v_{h}$ is linear. Clearly, when $\delta_{K}$ is a bubble function,

$$
\begin{aligned}
\varepsilon\left(\nabla u_{h}, \nabla\left(\widetilde{\delta} \mathbf{b} \cdot \nabla v_{h}\right)\right) & =\sum_{K} \varepsilon\left(\nabla u_{h}, \nabla\left(\delta_{K} \mathbf{b} \cdot \nabla v_{h}\right)\right)_{K} \\
& =\varepsilon \sum_{K} \int_{\partial K} \nabla u_{h} \cdot \mathbf{n} \delta_{K} \mathbf{b} \cdot \nabla v_{h} \\
& -\varepsilon \sum_{K} \int_{K}\left(\nabla \cdot \nabla u_{h}\right) \delta_{K} \mathbf{b} \cdot \nabla v_{h} \\
& =\varepsilon \sum_{K} \int_{\partial K} \nabla u_{h} \cdot \mathbf{n} \delta_{K} \mathbf{b} \cdot \nabla v_{h} \equiv 0,
\end{aligned}
$$

since $\delta_{K}$ vanishes on the boundary of $K$. Thus (22) can be written as

$$
\begin{aligned}
\varepsilon & \left(\nabla u_{h}, \nabla\left(v_{h}+\widetilde{\delta} \mathbf{b} \cdot \nabla v_{h}\right)\right)+\left(\mathbf{b} \cdot \nabla u_{h}+c u_{h}, v_{h}+\widetilde{\delta} \mathbf{b} \cdot \nabla v_{h}\right) \\
& =\left(f, v_{h}+\widetilde{\delta} \mathbf{b} \cdot \nabla v_{h}\right) .
\end{aligned}
$$

Then SUPG with bubble stabilization coefficients is classified into the consistent Petrov-Galerkin formulation.

Moreover, in general, piecewise constants $\delta_{K}$ in SUPG make the test function $v_{h}+\delta_{K} \mathbf{b} \cdot \nabla v_{h}$ discontinuous. However, the test functions are continuous in the case of bubble stabilization coefficients and the consequent test space $W_{h}$ is contained in $H_{0}^{1}(\Omega)$. In this case, SUPG with bubble stabilization coefficients reads as follows.

Find $u_{h} \in V_{h}$, such that for all $w_{h} \in W_{h}$,

$$
a\left(u_{h}, w_{h}\right)=\left(f, w_{h}\right) \text {, }
$$

where

$$
W_{h}:=\left\{w_{h} \in C(\bar{\Omega}):\left.w_{h}\right|_{K}=\left.v_{h}\right|_{K}+\left.\delta_{K} \mathbf{b} \cdot \nabla v_{h}\right|_{K}, v_{h} \in V_{h}\right\} .
$$

On the other hand, SUPG with bubble stabilization coefficients is gradually close to the FEM in the same space $H_{0}^{1}(\Omega)$ as $C_{K}^{*} \rightarrow 0$ for any $\in \mathscr{T}_{h}$.

In a word, SUPG with bubble stabilization coefficients inherits the advantages of SUPG and constructs the relation between FEM and SUPG in consistent Petrov-Galerkin formulations.

\section{Numerical Experiments}

In this section we give numerical results that appear to support our theoretical results. Errors and convergence rates for our numerical scheme are presented. All calculations are carried out by using Intel visual Fortran 11. The discrete problems are solved by using a version of Pardiso solver (see $[10,11])$.

For the computations we have chosen $\delta_{K}=60.0 h \lambda_{1} \lambda_{2} \lambda_{3}$ in SUPG with bubble stabilization coefficients and $\delta_{K}=1.0 \mathrm{~h}$ in SUPG. We set $\Omega=[0,1]^{2}$ and calculate the errors and convergence rates in the subdomain $\Omega_{C}$ away from layers for Tables 1-16. For parabolic Problems 1 and $2, \Omega_{C}=[0,1 / 2] \times$ $[1 / 3,2 / 3]$. For exponential Problems 3 and $4, \Omega_{C}=[0,1 / 2] \times$ $[0,1 / 2]$. The errors in Tables $17-20$ are calculated in the whole domain $\Omega$. In the following we only list the results of the case of $\varepsilon=10^{-2}$ and $\varepsilon=10^{-8}$ since the comparison results between the two methods of other cases like $\varepsilon=10^{-4}, \varepsilon=$ $10^{-6}$, and $\varepsilon=10^{-10}$ are similar.

\section{Problem 1. One has}

$$
\begin{aligned}
-\varepsilon \Delta u+(2-x) u_{x}+\frac{3}{2} u & =f \quad(x, y) \in \Omega, \\
u & =0 \quad(x, y) \in \partial \Omega,
\end{aligned}
$$

where $f$ is chosen such that the solution $u$ is

$$
\begin{aligned}
u(x, y)= & \left(\sin \frac{\pi}{2} x-\frac{e^{-((1-x) / \varepsilon)}-e^{-(1 / \varepsilon)}}{1-e^{-(1 / \varepsilon)}}\right) \\
& \times \frac{\left(1-e^{-(y / \sqrt{\varepsilon})}\right)\left(1-e^{-((1-y) / \sqrt{\varepsilon})}\right)}{1-e^{-1 / \sqrt{\varepsilon}}} .
\end{aligned}
$$

Problem 2. One has

$$
\begin{aligned}
-\varepsilon \Delta u+u_{x}+u & =x(1-x)+y(1-y), \quad(x, y) \in \Omega, \\
u & =0 \quad(x, y) \in \partial \Omega .
\end{aligned}
$$

Problem 3. One has

$$
\begin{aligned}
-\varepsilon \Delta u+2 u_{x}+3 u_{y}+u=f & (x, y) \in \Omega, \\
u=0 & (x, y) \in \partial \Omega,
\end{aligned}
$$

where $f$ is chosen such that the solution $u$ is

$$
\begin{aligned}
u(x, y)= & 2 \sin x\left(1-\exp \left(\frac{-2(1-x)}{\varepsilon}\right)\right) \\
& \times y^{2}\left(1-\exp \left(\frac{-(1-y)}{\varepsilon}\right)\right) .
\end{aligned}
$$

Problem 4. One has

$$
\begin{gathered}
-\varepsilon \Delta u+2 u_{x}+3 u_{y}+u=x(1-x)+y(1-y) \quad(x, y) \in \Omega, \\
u=0 \quad(x, y) \in \partial \Omega .
\end{gathered}
$$

From the above tables it is shown that the errors and convergence rates of SUPG with bubble stabilization coefficients and standard SUPG in the maximum norm, in the $L^{2}$ norm, and in the SD norm are similar not only in the subdomain away from layers but also in the global sense. These results illustrate that SUPG with bubble stabilization coefficients also has good stability properties and high accuracy as standard SUPG. 
TABle 1: $\varepsilon=10^{-8}$, Problem 1 (comparison of error).

\begin{tabular}{|c|c|c|c|c|c|c|}
\hline \multirow{2}{*}{$N$} & \multicolumn{2}{|c|}{$L^{\infty}$ norm } & \multicolumn{2}{|c|}{$L^{2}$ norm } & \multicolumn{2}{|c|}{ SD norm } \\
\hline & Bubble SUPG & SUPG & Bubble SUPG & SUPG & Bubble SUPG & SUPG \\
\hline 6 & $4.10 \times 10^{-2}$ & $4.13 \times 10^{-2}$ & $2.59 \times 10^{-2}$ & $2.60 \times 10^{-2}$ & $2.59 \times 10^{-2}$ & $2.6 \times 10^{-2}$ \\
\hline 12 & $6.30 \times 10^{-3}$ & $6.35 \times 10^{-3}$ & $3.32 \times 10^{-3}$ & $3.34 \times 10^{-3}$ & $3.33 \times 10^{-3}$ & $3.34 \times 10^{-3}$ \\
\hline 24 & $3.53 \times 10^{-4}$ & $3.45 \times 10^{-4}$ & $1.32 \times 10^{-4}$ & $1.33 \times 10^{-4}$ & $1.32 \times 10^{-4}$ & $1.33 \times 10^{-4}$ \\
\hline 48 & $1.14 \times 10^{-4}$ & $1.13 \times 10^{-4}$ & $2.00 \times 10^{-5}$ & $2.00 \times 10^{-5}$ & $2.00 \times 10^{-5}$ & $2.01 \times 10^{-5}$ \\
\hline 96 & $3.11 \times 10^{-5}$ & $3.10 \times 10^{-5}$ & $4.74 \times 10^{-6}$ & $4.75 \times 10^{-6}$ & $4.75 \times 10^{-6}$ & $4.75 \times 10^{-6}$ \\
\hline 192 & $8.15 \times 10^{-6}$ & $8.13 \times 10^{-6}$ & $1.16 \times 10^{-6}$ & $1.16 \times 10^{-6}$ & $1.16 \times 10^{-6}$ & $1.16 \times 10^{-6}$ \\
\hline
\end{tabular}

TABLe 2: $\varepsilon=10^{-8}$, Problem 1 (comparison of convergence rate).

\begin{tabular}{lcccccc}
\hline$N$ & \multicolumn{2}{c}{$L^{\infty}$ norm } & \multicolumn{2}{c}{$L^{2}$ norm } & \multicolumn{2}{c}{ SD norm } \\
& Bubble SUPG & SUPG & Bubble SUPG & SUPG & Bubble SUPG & SUPG \\
\hline 6 & 2.70 & 2.70 & 2.95 & 2.96 & 4.65 & 4.96 \\
12 & 4.16 & 4.20 & 4.66 & 2.73 & 2.73 & 2.73 \\
24 & 1.63 & 1.61 & 2.73 & 2.08 & 2.07 & 2.06 \\
48 & 1.87 & 1.87 & 2.07 & 2.04 & 2.03 \\
96 & 1.93 & 1.93 & 2.03 & & 2.03 \\
\hline
\end{tabular}

TABle 3: $\varepsilon=10^{-2}$, Problem 1 (comparison of error).

\begin{tabular}{|c|c|c|c|c|c|c|}
\hline \multirow{2}{*}{$N$} & \multicolumn{2}{|c|}{$L^{\infty}$ norm } & \multicolumn{2}{|c|}{$L^{2}$ norm } & \multicolumn{2}{|c|}{ SD norm } \\
\hline & Bubble SUPG & SUPG & Bubble SUPG & SUPG & Bubble SUPG & SUPG \\
\hline 6 & $3.63 \times 10^{-2}$ & $3.63 \times 10^{-2}$ & $2.75 \times 10^{-2}$ & $2.73 \times 10^{-2}$ & $3.02 \times 10^{-2}$ & $3.01 \times 10^{-2}$ \\
\hline 12 & $9.03 \times 10^{-3}$ & $9.07 \times 10^{-3}$ & $4.63 \times 10^{-3}$ & $4.64 \times 10^{-3}$ & $6.23 \times 10^{-3}$ & $6.23 \times 10^{-3}$ \\
\hline 24 & $9.72 \times 10^{-4}$ & $9.85 \times 10^{-4}$ & $4.71 \times 10^{-4}$ & $4.74 \times 10^{-4}$ & $1.36 \times 10^{-3}$ & $1.36 \times 10^{-3}$ \\
\hline 48 & $3.96 \times 10^{-4}$ & $3.98 \times 10^{-4}$ & $1.63 \times 10^{-4}$ & $1.64 \times 10^{-4}$ & $6.40 \times 10^{-4}$ & $6.39 \times 10^{-4}$ \\
\hline 96 & $2.58 \times 10^{-4}$ & $2.59 \times 10^{-4}$ & $8.24 \times 10^{-5}$ & $8.25 \times 10^{-5}$ & $3.20 \times 10^{-4}$ & $3.20 \times 10^{-4}$ \\
\hline 192 & $1.47 \times 10^{-4}$ & $1.47 \times 10^{-4}$ & $4.19 \times 10^{-5}$ & $4.19 \times 10^{-5}$ & $1.61 \times 10^{-4}$ & $1.61 \times 10^{-4}$ \\
\hline
\end{tabular}

TABLE 4: $\varepsilon=10^{-2}$, Problem 1 (comparison of convergence rate).

\begin{tabular}{|c|c|c|c|c|c|c|}
\hline \multirow{2}{*}{$N$} & \multicolumn{2}{|c|}{$L^{\infty}$ norm } & \multicolumn{2}{|c|}{$L^{2}$ norm } & \multicolumn{2}{|c|}{ SD norm } \\
\hline & Bubble SUPG & SUPG & Bubble SUPG & SUPG & Bubble SUPG & SUPG \\
\hline 6 & 2.01 & 2.00 & 2.57 & 2.56 & 2.28 & 2.27 \\
\hline 12 & 3.22 & 3.20 & 3.30 & 3.29 & 2.20 & 2.20 \\
\hline 24 & 1.30 & 1.31 & 1.53 & 1.53 & 1.09 & 1.09 \\
\hline 48 & 0.62 & 0.62 & 0.99 & 0.99 & 1.00 & 1.00 \\
\hline 96 & 0.82 & 0.82 & 0.98 & 0.98 & 1.00 & 1.00 \\
\hline
\end{tabular}

TABLE 5: $\varepsilon=10^{-8}$, Problem 2 (comparison of error).

\begin{tabular}{|c|c|c|c|c|c|c|}
\hline \multirow{2}{*}{$N$} & \multicolumn{2}{|c|}{$L^{\infty}$ norm } & \multicolumn{2}{|c|}{$L^{2}$ norm } & \multicolumn{2}{|c|}{ SD norm } \\
\hline & Bubble SUPG & SUPG & Bubble SUPG & SUPG & Bubble SUPG & SUPG \\
\hline 6 & $4.99 \times 10^{-3}$ & $5.01 \times 10^{-3}$ & $5.80 \times 10^{-3}$ & $5.82 \times 10^{-3}$ & $8.81 \times 10^{-3}$ & $9.23 \times 10^{-3}$ \\
\hline 12 & $6.04 \times 10^{-4}$ & $6.04 \times 10^{-4}$ & $5.63 \times 10^{-4}$ & $5.64 \times 10^{-4}$ & $1.33 \times 10^{-3}$ & $1.42 \times 10^{-3}$ \\
\hline 24 & $2.37 \times 10^{-4}$ & $2.36 \times 10^{-4}$ & $5.54 \times 10^{-5}$ & $5.54 \times 10^{-5}$ & $2.93 \times 10^{-4}$ & $3.16 \times 10^{-4}$ \\
\hline 48 & $6.59 \times 10^{-5}$ & $6.59 \times 10^{-5}$ & $1.43 \times 10^{-5}$ & $1.43 \times 10^{-5}$ & $1.02 \times 10^{-4}$ & $1.10 \times 10^{-4}$ \\
\hline 96 & $1.73 \times 10^{-5}$ & $1.73 \times 10^{-5}$ & $3.65 \times 10^{-6}$ & $3.65 \times 10^{-6}$ & $3.60 \times 10^{-5}$ & $3.88 \times 10^{-5}$ \\
\hline
\end{tabular}

TABLE 6: $\varepsilon=10^{-8}$, Problem 2 (comparison of convergence rate).

\begin{tabular}{lccccc}
\hline$N$ & \multicolumn{2}{c}{$L^{\infty}$ norm } & \multicolumn{2}{c}{$L^{2}$ norm } & \multicolumn{2}{c}{ SD norm } \\
& Bubble SUPG & SUPG & Bubble SUPG & SUPG & Bubble SUPG \\
\hline 6 & 3.05 & 3.05 & 3.37 & 3.37 & 2.72 \\
12 & 1.35 & 1.35 & 3.34 & 3.35 & 2.18 \\
24 & 1.84 & 1.84 & 1.95 & 1.95 & 1.52 \\
48 & 1.93 & 1.93 & 1.97 & 1.97 & 1.52 \\
\hline
\end{tabular}


TABLe 7: $\varepsilon=10^{-2}$, Problem 2 (comparison of error).

\begin{tabular}{|c|c|c|c|c|c|c|}
\hline \multirow{2}{*}{$N$} & \multicolumn{2}{|c|}{$L^{\infty}$ norm } & \multicolumn{2}{|c|}{$L^{2}$ norm } & \multicolumn{2}{|c|}{ SD norm } \\
\hline & Bubble SUPG & SUPG & Bubble SUPG & SUPG & Bubble SUPG & SUPG \\
\hline 6 & $6.67 \times 10^{-3}$ & $6.69 \times 10^{-3}$ & $6.12 \times 10^{-3}$ & $6.13 \times 10^{-3}$ & $9.36 \times 10^{-3}$ & $9.75 \times 10^{-3}$ \\
\hline 12 & $1.32 \times 10^{-3}$ & $1.32 \times 10^{-3}$ & $9.23 \times 10^{-4}$ & $9.25 \times 10^{-4}$ & $1.94 \times 10^{-3}$ & $2.03 \times 10^{-3}$ \\
\hline 24 & $1.72 \times 10^{-4}$ & $1.72 \times 10^{-4}$ & $1.07 \times 10^{-4}$ & $1.07 \times 10^{-4}$ & $4.55 \times 10^{-4}$ & $4.69 \times 10^{-4}$ \\
\hline 48 & $1.09 \times 10^{-4}$ & $1.09 \times 10^{-4}$ & $4.56 \times 10^{-5}$ & $4.57 \times 10^{-5}$ & $1.98 \times 10^{-4}$ & $2.01 \times 10^{-4}$ \\
\hline 96 & $7.14 \times 10^{-5}$ & $7.14 \times 10^{-5}$ & $2.45 \times 10^{-5}$ & $2.45 \times 10^{-5}$ & $9.28 \times 10^{-5}$ & $9.39 \times 10^{-5}$ \\
\hline
\end{tabular}

TABLE 8: $\varepsilon=10^{-2}$, Problem 2 (comparison of convergence rate).

\begin{tabular}{lccccc}
\hline \multirow{2}{*}{$\begin{array}{c}L^{\infty} \text { norm } \\
\text { Bubble SUPG }\end{array}$} & SUPG & Bubble SUPG & SUPG & \multicolumn{2}{c}{$\begin{array}{c}L^{2} \text { norm norm } \\
\text { Bubble SUPG }\end{array}$} \\
\hline 6 & 2.34 & 2.34 & 2.73 & 2.73 & 2.27 \\
12 & 2.94 & 2.94 & 3.11 & 3.12 & 2.09 \\
24 & 0.66 & 0.67 & 1.22 & 1.22 & 1.20 \\
48 & 0.60 & 0.60 & 0.90 & 0.90 & 1.26 \\
\hline
\end{tabular}

TABle 9: $\varepsilon=10^{-8}$, Problem 3 (comparison of error).

\begin{tabular}{|c|c|c|c|c|c|c|}
\hline \multirow{2}{*}{$N$} & \multicolumn{2}{|c|}{$L^{\infty}$ norm } & \multicolumn{2}{|c|}{$L^{2}$ norm } & \multicolumn{2}{|c|}{ SD norm } \\
\hline & Bubble SUPG & SUPG & Bubble SUPG & SUPG & Bubble SUPG & SUPG \\
\hline 6 & $2.70 \times 10^{-1}$ & $2.70 \times 10^{-1}$ & $5.66 \times 10^{-2}$ & $5.66 \times 10^{-2}$ & $3.27 \times 10^{-1}$ & $3.27 \times 10^{-1}$ \\
\hline 12 & $9.15 \times 10^{-2}$ & $9.15 \times 10^{-2}$ & $1.51 \times 10^{-2}$ & $1.51 \times 10^{-2}$ & $6.40 \times 10^{-2}$ & $6.43 \times 10^{-2}$ \\
\hline 24 & $6.60 \times 10^{-3}$ & $6.60 \times 10^{-3}$ & $8.04 \times 10^{-4}$ & $8.04 \times 10^{-4}$ & $6.35 \times 10^{-3}$ & $6.71 \times 10^{-3}$ \\
\hline 48 & $1.62 \times 10^{-5}$ & $1.62 \times 10^{-5}$ & $1.09 \times 10^{-5}$ & $1.09 \times 10^{-5}$ & $2.13 \times 10^{-3}$ & $2.26 \times 10^{-3}$ \\
\hline 96 & $3.84 \times 10^{-6}$ & $3.85 \times 10^{-6}$ & $2.56 \times 10^{-6}$ & $2.56 \times 10^{-6}$ & $7.53 \times 10^{-4}$ & $7.80 \times 10^{-4}$ \\
\hline 192 & $9.51 \times 10^{-7}$ & $9.52 \times 10^{-7}$ & $6.38 \times 10^{-7}$ & $6.38 \times 10^{-7}$ & $2.66 \times 10^{-4}$ & $2.83 \times 10^{-4}$ \\
\hline
\end{tabular}

TABLE 10: $\varepsilon=10^{-8}$, Problem 3 (comparison of convergence rate).

\begin{tabular}{|c|c|c|c|c|c|c|}
\hline \multirow{2}{*}{$N$} & \multicolumn{2}{|c|}{$L^{\infty}$ norm } & \multicolumn{2}{|c|}{$L^{2}$ norm } & \multicolumn{2}{|c|}{ SD norm } \\
\hline & Bubble SUPG & SUPG & Bubble SUPG & SUPG & Bubble SUPG & SUPG \\
\hline 6 & 1.56 & 1.56 & 1.91 & 1.91 & 2.35 & 2.35 \\
\hline 12 & 3.79 & 3.79 & 4.23 & 4.23 & 3.33 & 3.26 \\
\hline 24 & 8.67 & 8.67 & 6.21 & 6.21 & 1.58 & 1.57 \\
\hline 48 & 2.08 & 2.07 & 2.08 & 2.08 & 1.50 & 1.50 \\
\hline 96 & 2.01 & 2.02 & 2.01 & 2.01 & 1.50 & 1.50 \\
\hline
\end{tabular}

TABLE 11: $\varepsilon=10^{-2}$, Problem 3 (comparison of error).

\begin{tabular}{|c|c|c|c|c|c|c|}
\hline \multirow{2}{*}{$N$} & \multicolumn{2}{|c|}{$L^{\infty}$ norm } & \multicolumn{2}{|c|}{$L^{2}$ norm } & \multicolumn{2}{|c|}{ SD norm } \\
\hline & Bubble SUPG & SUPG & Bubble SUPG & SUPG & Bubble SUPG & SUPG \\
\hline 6 & $1.66 \times 10^{-1}$ & $1.48 \times 10^{-1}$ & $3.65 \times 10^{-2}$ & $3.30 \times 10^{-2}$ & $2.14 \times 10^{-1}$ & $1.96 \times 10^{-1}$ \\
\hline 12 & $4.07 \times 10^{-2}$ & $3.28 \times 10^{-2}$ & $7.07 \times 10^{-3}$ & $5.83 \times 10^{-3}$ & $3.65 \times 10^{-2}$ & $3.38 \times 10^{-2}$ \\
\hline 24 & $2.59 \times 10^{-3}$ & $2.23 \times 10^{-3}$ & $3.06 \times 10^{-4}$ & $2.63 \times 10^{-4}$ & $6.13 \times 10^{-3}$ & $6.49 \times 10^{-3}$ \\
\hline 48 & $1.41 \times 10^{-4}$ & $1.42 \times 10^{-4}$ & $4.02 \times 10^{-5}$ & $4.02 \times 10^{-5}$ & $2.19 \times 10^{-3}$ & $2.31 \times 10^{-3}$ \\
\hline 96 & $7.77 \times 10^{-5}$ & $7.77 \times 10^{-5}$ & $2.01 \times 10^{-5}$ & $2.01 \times 10^{-5}$ & $7.91 \times 10^{-4}$ & $8.35 \times 10^{-4}$ \\
\hline 192 & $3.85 \times 10^{-5}$ & $3.85 \times 10^{-5}$ & $9.98 \times 10^{-6}$ & $9.98 \times 10^{-6}$ & $2.92 \times 10^{-4}$ & $3.07 \times 10^{-4}$ \\
\hline
\end{tabular}

TABle 12: $\varepsilon=10^{-2}$, Problem 3 (comparison of convergence rate).

\begin{tabular}{|c|c|c|c|c|c|c|}
\hline \multirow{2}{*}{$N$} & \multicolumn{2}{|c|}{$L^{\infty}$ norm } & \multicolumn{2}{|c|}{$L^{2}$ norm } & \multicolumn{2}{|c|}{ SD norm } \\
\hline & Bubble SUPG & SUPG & Bubble SUPG & SUPG & Bubble SUPG & SUPG \\
\hline 6 & 2.03 & 2.18 & 2.37 & 2.50 & 2.55 & 2.54 \\
\hline 12 & 3.97 & 3.88 & 4.53 & 4.47 & 2.57 & 2.38 \\
\hline 24 & 4.20 & 3.97 & 2.93 & 2.71 & 1.49 & 1.49 \\
\hline 48 & 0.86 & 0.87 & 1.00 & 1.00 & 1.47 & 1.47 \\
\hline 96 & 1.01 & 1.01 & 1.01 & 1.01 & 1.44 & 1.44 \\
\hline
\end{tabular}


TABLE 13: $\varepsilon=10^{-8}$, Problem 4 (comparison of error).

\begin{tabular}{|c|c|c|c|c|c|c|}
\hline \multirow{2}{*}{$N$} & \multicolumn{2}{|c|}{$L^{\infty}$ norm } & \multicolumn{2}{|c|}{$L^{2}$ norm } & \multicolumn{2}{|c|}{ SD norm } \\
\hline & Bubble SUPG & SUPG & Bubble SUPG & SUPG & Bubble SUPG & SUPG \\
\hline 6 & $1.24 \times 10^{-2}$ & $1.24 \times 10^{-2}$ & $4.31 \times 10^{-3}$ & $4.31 \times 10^{-3}$ & $2.11 \times 10^{-2}$ & $2.11 \times 10^{-2}$ \\
\hline 12 & $8.76 \times 10^{-3}$ & $8.76 \times 10^{-3}$ & $2.35 \times 10^{-3}$ & $2.35 \times 10^{-3}$ & $9.09 \times 10^{-3}$ & $9.09 \times 10^{-3}$ \\
\hline 24 & $1.54 \times 10^{-3}$ & $1.54 \times 10^{-3}$ & $3.20 \times 10^{-4}$ & $3.20 \times 10^{-4}$ & $2.04 \times 10^{-3}$ & $2.04 \times 10^{-3}$ \\
\hline 48 & $1.06 \times 10^{-4}$ & $1.06 \times 10^{-4}$ & $1.78 \times 10^{-5}$ & $1.78 \times 10^{-5}$ & $6.65 \times 10^{-4}$ & $6.65 \times 10^{-4}$ \\
\hline 96 & $2.59 \times 10^{-5}$ & $2.59 \times 10^{-5}$ & $4.06 \times 10^{-6}$ & $4.06 \times 10^{-6}$ & $2.35 \times 10^{-4}$ & $2.35 \times 10^{-4}$ \\
\hline
\end{tabular}

TABle 14: $\varepsilon=10^{-8}$, Problem 4 (comparison of convergence rate).

\begin{tabular}{lccccc}
\hline$N$ & \multicolumn{2}{c}{$L^{\infty}$ norm } & \multicolumn{2}{c}{$L^{2}$ norm } & \multicolumn{2}{c}{ SD norm } \\
& Bubble SUPG & SUPG & Bubble SUPG & SUPG & Bubble SUPG \\
\hline 6 & 0.50 & 0.50 & 0.87 & 0.87 & 1.22 \\
12 & 2.51 & 2.51 & 2.88 & 2.88 & 2.16 \\
24 & 3.85 & 3.85 & 4.16 & 4.16 & 1.22 \\
48 & 2.04 & 2.04 & 2.13 & 2.13 & 1.62 \\
\hline
\end{tabular}

TABle 15: $\varepsilon=10^{-2}$, Problem 4 (comparison of error).

\begin{tabular}{|c|c|c|c|c|c|c|}
\hline \multirow{2}{*}{$N$} & \multicolumn{2}{|c|}{$L^{\infty}$ norm } & \multicolumn{2}{|c|}{$L^{2}$ norm } & \multicolumn{2}{|c|}{ SD norm } \\
\hline & Bubble SUPG & SUPG & Bubble SUPG & SUPG & Bubble SUPG & SUPG \\
\hline 6 & $1.21 \times 10^{-2}$ & $1.21 \times 10^{-2}$ & $4.23 \times 10^{-3}$ & $4.23 \times 10^{-3}$ & $2.08 \times 10^{-2}$ & $2.08 \times 10^{-2}$ \\
\hline 12 & $8.77 \times 10^{-3}$ & $8.77 \times 10^{-3}$ & $2.34 \times 10^{-3}$ & $2.34 \times 10^{-3}$ & $9.07 \times 10^{-3}$ & $9.07 \times 10^{-3}$ \\
\hline 24 & $1.62 \times 10^{-3}$ & $1.62 \times 10^{-3}$ & $3.56 \times 10^{-4}$ & $3.56 \times 10^{-4}$ & $2.05 \times 10^{-3}$ & $2.05 \times 10^{-3}$ \\
\hline 48 & $1.14 \times 10^{-4}$ & $1.14 \times 10^{-4}$ & $2.59 \times 10^{-5}$ & $2.59 \times 10^{-5}$ & $6.63 \times 10^{-4}$ & $6.63 \times 10^{-4}$ \\
\hline 96 & $2.73 \times 10^{-5}$ & $2.73 \times 10^{-5}$ & $8.23 \times 10^{-6}$ & $8.23 \times 10^{-6}$ & $2.41 \times 10^{-4}$ & $2.41 \times 10^{-4}$ \\
\hline
\end{tabular}

TABle 16: $\varepsilon=10^{-2}$, Problem 4 (comparison of convergence rate).

\begin{tabular}{lccccc}
\hline \multirow{2}{*}{$\begin{array}{c}L^{\infty} \text { norm } \\
\text { Bubble SUPG }\end{array}$} & SUPG & Bubble SUPG & SUPG & \multicolumn{2}{c}{$\begin{array}{c}L^{2} \text { norm norm } \\
\text { Bubble SUPG }\end{array}$} \\
\hline 6 & 0.47 & 0.47 & 0.85 & 0.85 & 1.20 \\
12 & 2.44 & 2.44 & 2.72 & 2.72 & 2.14 \\
24 & 3.82 & 3.82 & 3.78 & 3.78 & 1.20 \\
48 & 2.06 & 2.06 & 1.65 & 1.65 & 1.63 \\
\hline
\end{tabular}

TABLe 17: $\varepsilon=10^{-8}$, Problem 1 (comparison of global error).

\begin{tabular}{lccccc}
\hline \multirow{2}{*}{$N$} & \multicolumn{2}{c}{$L^{\infty}$ norm } & \multicolumn{2}{c}{$L^{2}$ norm } & \multicolumn{2}{c}{ SD norm } \\
& Bubble SUPG & SUPG & Bubble SUPG & SUPG & Bubble SUPG \\
\hline 6 & $3.18 \times 10^{-1}$ & $3.19 \times 10^{-1}$ & $3.72 \times 10^{-1}$ & $3.73 \times 10^{-1}$ & 1.44 \\
12 & $3.31 \times 10^{-1}$ & $3.32 \times 10^{-1}$ & $2.64 \times 10^{-1}$ & $2.64 \times 10^{-1}$ & 1.44 \\
24 & $3.38 \times 10^{-1}$ & $3.38 \times 10^{-1}$ & $1.86 \times 10^{-1}$ & $1.86 \times 10^{-1}$ & 1.42 \\
48 & $3.41 \times 10^{-1}$ & $3.41 \times 10^{-1}$ & $1.31 \times 10^{-1}$ & $1.31 \times 10^{-1}$ & 1.41 \\
96 & $3.42 \times 10^{-1}$ & $3.42 \times 10^{-1}$ & $9.29 \times 10^{-2}$ & $9.29 \times 10^{-2}$ & 1.41 \\
192 & $3.43 \times 10^{-1}$ & $3.43 \times 10^{-1}$ & $6.54 \times 10^{-2}$ & $6.54 \times 10^{-2}$ & 1.41 \\
\hline
\end{tabular}

TABLE 18: $\varepsilon=10^{-8}$, Problem 2 (comparison of global error).

\begin{tabular}{|c|c|c|c|c|c|c|}
\hline \multirow{2}{*}{$N$} & \multicolumn{2}{|c|}{$L^{\infty}$ norm } & \multicolumn{2}{|c|}{$L^{2}$ norm } & \multicolumn{2}{|c|}{ SD norm } \\
\hline & Bubble SUPG & SUPG & Bubble SUPG & SUPG & Bubble SUPG & SUPG \\
\hline 6 & $6.60 \times 10^{-2}$ & $6.60 \times 10^{-2}$ & $3.13 \times 10^{-2}$ & $3.14 \times 10^{-2}$ & $6.61 \times 10^{-2}$ & $7.02 \times 10^{-2}$ \\
\hline 12 & $6.56 \times 10^{-2}$ & $6.56 \times 10^{-2}$ & $2.16 \times 10^{-2}$ & $2.16 \times 10^{-2}$ & $5.78 \times 10^{-2}$ & $6.17 \times 10^{-2}$ \\
\hline 24 & $6.55 \times 10^{-2}$ & $6.55 \times 10^{-2}$ & $1.52 \times 10^{-2}$ & $1.52 \times 10^{-2}$ & $5.42 \times 10^{-2}$ & $5.81 \times 10^{-2}$ \\
\hline 48 & $6.55 \times 10^{-2}$ & $6.55 \times 10^{-2}$ & $1.08 \times 10^{-2}$ & $1.08 \times 10^{-2}$ & $5.26 \times 10^{-2}$ & $5.65 \times 10^{-2}$ \\
\hline 96 & $6.54 \times 10^{-2}$ & $6.54 \times 10^{-2}$ & $7.63 \times 10^{-3}$ & $7.63 \times 10^{-3}$ & $5.18 \times 10^{-2}$ & $5.58 \times 10^{-2}$ \\
\hline
\end{tabular}


TABle 19: $\varepsilon=10^{-8}$, Problem 3 (comparison of global error).

\begin{tabular}{lccccc}
\hline$N$ & \multicolumn{2}{c}{$L^{\infty}$ norm } & \multicolumn{2}{c}{$L^{2}$ norm } & \multicolumn{2}{c}{ SD norm } \\
& Bubble SUPG & SUPG & Bubble SUPG & SUPG & Bubble SUPG \\
\hline 6 & 1.16 & 1.16 & $4.84 \times 10^{-1}$ & $4.84 \times 10^{-1}$ & 1.47 \\
12 & 1.25 & 1.25 & $3.73 \times 10^{-1}$ & $3.73 \times 10^{-1}$ & 1.20 \\
24 & 1.28 & 1.28 & $2.66 \times 10^{-1}$ & $2.66 \times 10^{-1}$ & 1.41 \\
48 & 1.29 & 1.29 & $1.88 \times 10^{-1}$ & $1.88 \times 10^{-1}$ & 1.41 \\
96 & 1.30 & 1.30 & $1.32 \times 10^{-1}$ & $1.32 \times 10^{-3}$ & 1.62 \\
\hline
\end{tabular}

TABLe 20: $\varepsilon=10^{-8}$, Problem 4 (comparison of global error).

\begin{tabular}{|c|c|c|c|c|c|c|}
\hline \multirow{2}{*}{$N$} & \multicolumn{2}{|c|}{$L^{\infty}$ norm } & \multicolumn{2}{|c|}{$L^{2}$ norm } & \multicolumn{2}{|c|}{ SD norm } \\
\hline & Bubble SUPG & SUPG & Bubble SUPG & SUPG & Bubble SUPG & SUPG \\
\hline 6 & $2.53 \times 10^{-2}$ & $2.53 \times 10^{-2}$ & $1.26 \times 10^{-2}$ & $1.26 \times 10^{-2}$ & $7.82 \times 10^{-2}$ & $7.82 \times 10^{-2}$ \\
\hline 12 & $2.97 \times 10^{-2}$ & $2.97 \times 10^{-2}$ & $1.27 \times 10^{-2}$ & $1.27 \times 10^{-2}$ & $7.18 \times 10^{-2}$ & $7.18 \times 10^{-2}$ \\
\hline 24 & $2.87 \times 10^{-2}$ & $2.87 \times 10^{-2}$ & $9.44 \times 10^{-3}$ & $9.44 \times 10^{-3}$ & $6.66 \times 10^{-2}$ & $6.66 \times 10^{-2}$ \\
\hline 48 & $2.79 \times 10^{-2}$ & $2.79 \times 10^{-2}$ & $6.60 \times 10^{-3}$ & $6.60 \times 10^{-3}$ & $6.43 \times 10^{-2}$ & $6.43 \times 10^{-2}$ \\
\hline 96 & $2.76 \times 10^{-2}$ & $2.76 \times 10^{-2}$ & $4.64 \times 10^{-3}$ & $4.64 \times 10^{-3}$ & $6.34 \times 10^{-2}$ & $6.34 \times 10^{-2}$ \\
\hline
\end{tabular}

\section{Conflict of Interests}

The authors declare that there is no conflict of interests regarding the publication of this paper.

\section{Acknowledgments}

This work was partially supported by Promotive Research Fund for Excellent Young and Middle-aged Scientists of Shandong Province (Grant no. BS2012SF012) and Independent Innovation Foundation of Shandong University (Grant no. 2012TS194).

\section{References}

[1] M. Stynes, "Steady-state convection-diffusion problems," Acta Numerica, vol. 14, pp. 445-508, 2005.

[2] H. G. Roos, M. Stynes, and L. Tobiska, Robust Numerical Methods for Singularly Perturbed Differential Equations: ConvectionDiffusion-Reaction and Flow Problems, Springer, Berlin, Germany, 2008.

[3] T. J. R. Hughes and A. Brooks, "A multidimensional upwind scheme with no crosswind diffusion," in Finite Element Methods for Convection Dominated Flows, T. J. R. Hughes, Ed., vol. 34 of $A M D$, pp. 19-35, American Society of Mechanical Engineers, Applied Mechanics Division, New York, NY, USA, 1979.

[4] V. John and P. Knobloch, "On spurious oscillations at layers diminishing (SOLD) methods for convection-diffusion equations-I. A review," Computer Methods in Applied Mechanics and Engineering, vol. 196, no. 17-20, pp. 2197-2215, 2007.

[5] E. Burman and A. Ern, "Nonlinear diffusion and discrete maximum principle for stabilized Galerkin approximations of the convection-diffusion-reaction equation," Computer Methods in Applied Mechanics and Engineering, vol. 191, no. 35, pp. 3833$3855,2002$.

[6] R. Becker and M. Braack, "A finite element pressure gradient stabilization for the Stokes equations based on local projections," Calcolo, vol. 38, no. 4, pp. 173-199, 2001.
[7] L. P. Franca and A. Russo, "Recovering SUPG using PetrovGalerkin formulations enriched with adjoint residual-free bubbles," Computer Methods in Applied Mechanics and Engineering, vol. 182, no. 3-4, pp. 333-339, 2000.

[8] T. J. R. Hughes, "Multiscale phenomena: Green's functions, the Dirichlet-to-Neumann formulation, subgrid scale models, bubbles and the origins of stabilized methods," Computer Methods in Applied Mechanics and Engineering, vol. 127, no. 1-4, pp. 387-401, 1995.

[9] P. G. Ciarlet, The Finite Element Method for Elliptic Problems, vol. 40 of Classics in Applied Mathematics, SIAM, Philadelphia, $\mathrm{Pa}$, USA, 2002.

[10] O. Schenk and K. N. Nävert, "Solving unsymmetric sparse systems of linear equations with PARDISO," Journal of Future Generation Computer Systems, vol. 20, pp. 475-487, 2004.

[11] O. Schenk and K. N. Nävert, "On fast factorization pivoting methods for symmetric indefinite systems," Electronic Transactions on Numerical Analysis, vol. 23, pp. 158-179, 2006. 


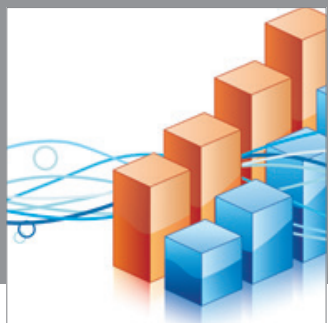

Advances in

Operations Research

mansans

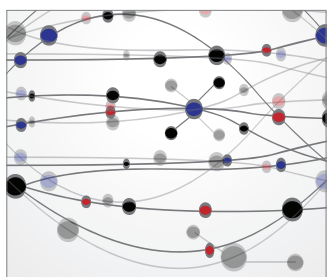

The Scientific World Journal
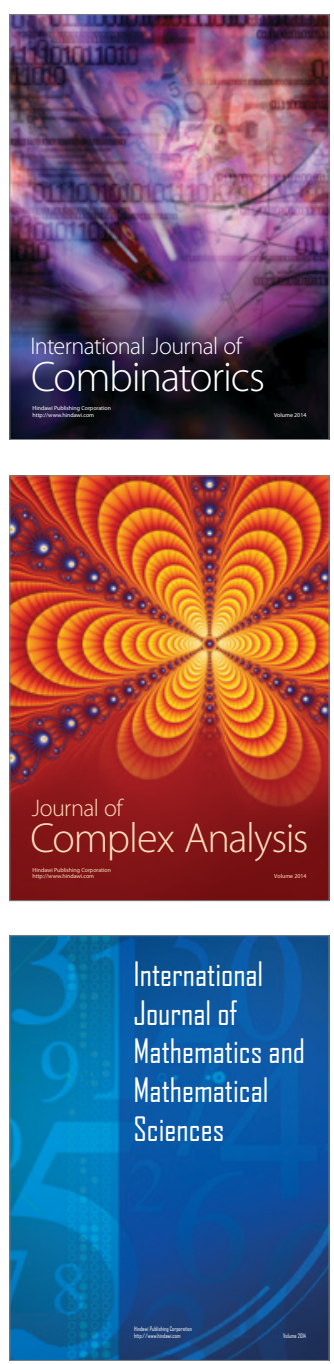
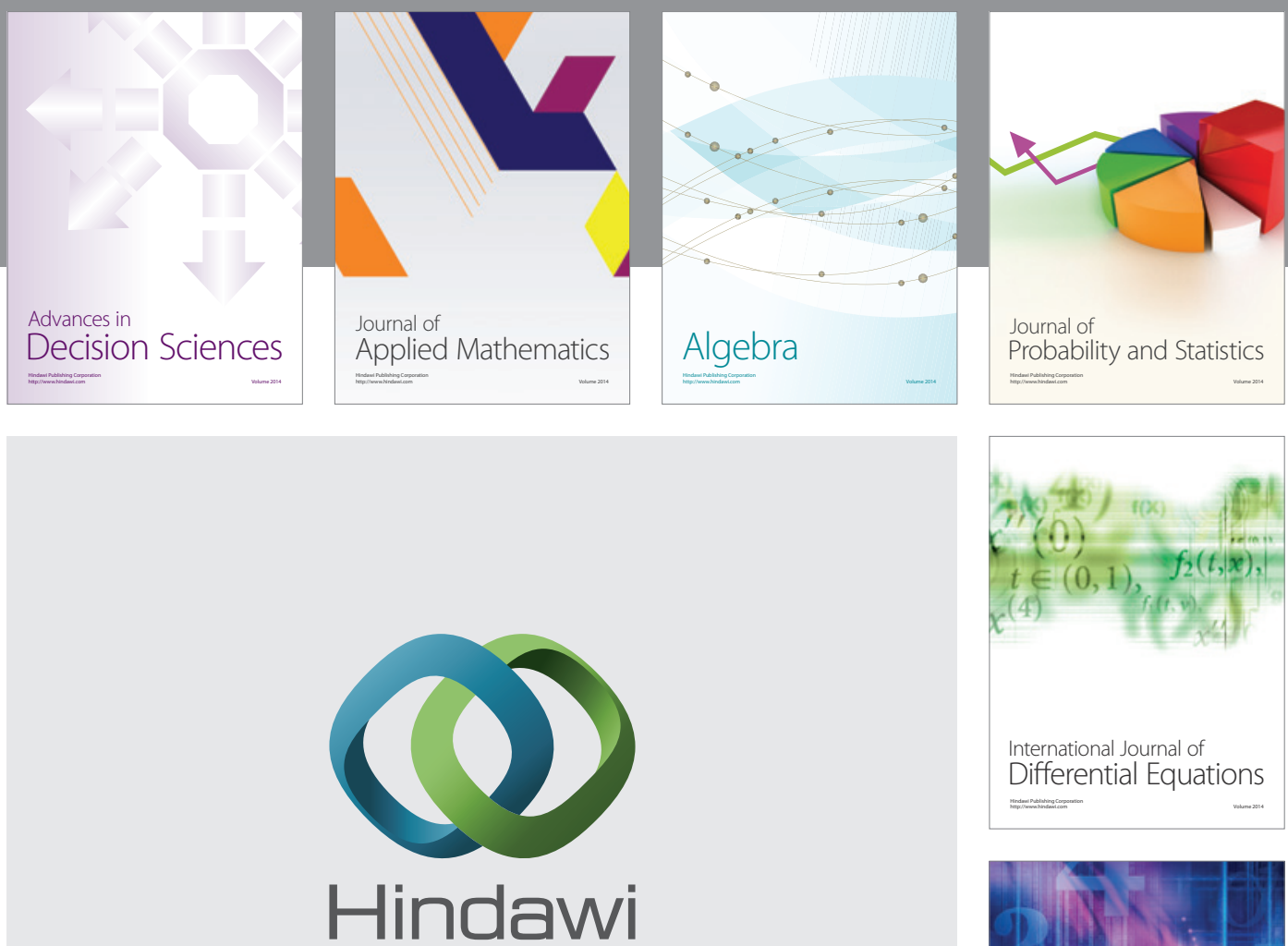

Submit your manuscripts at http://www.hindawi.com
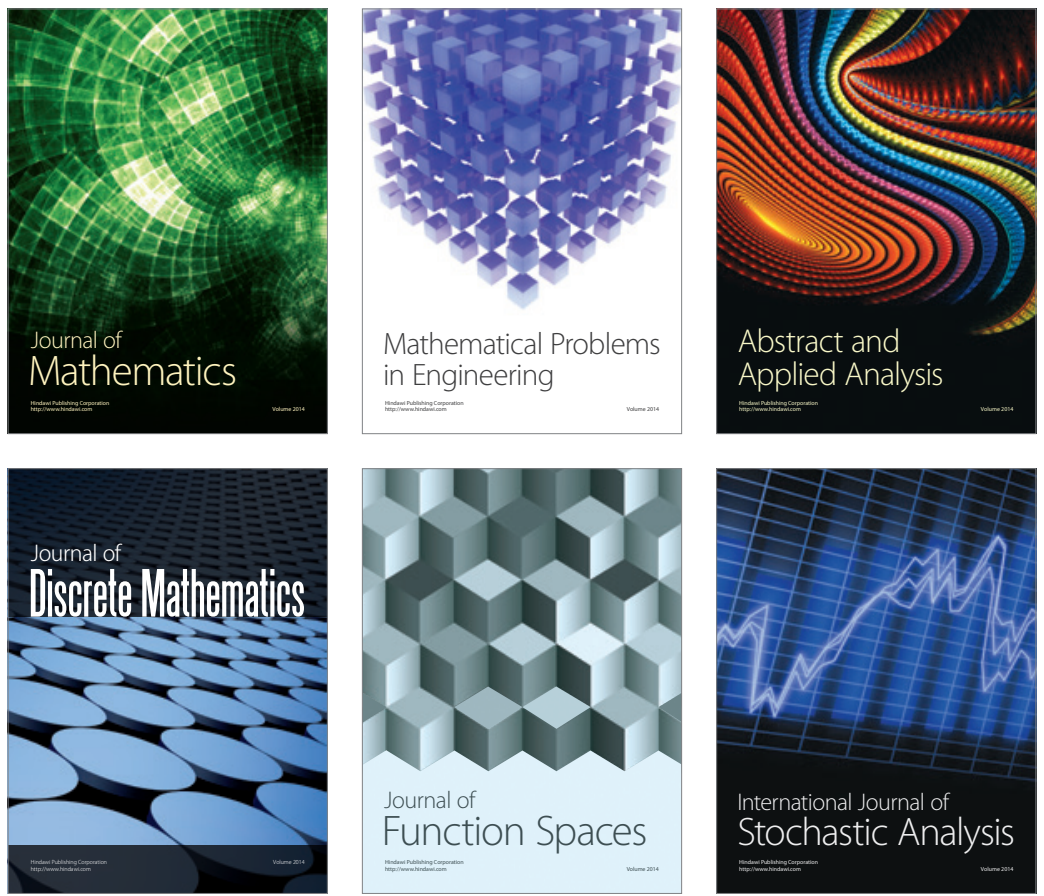

Journal of

Function Spaces

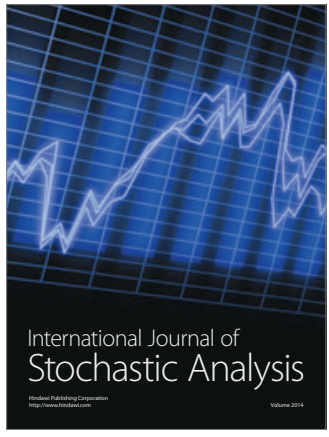

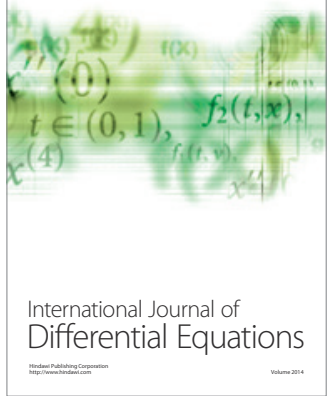
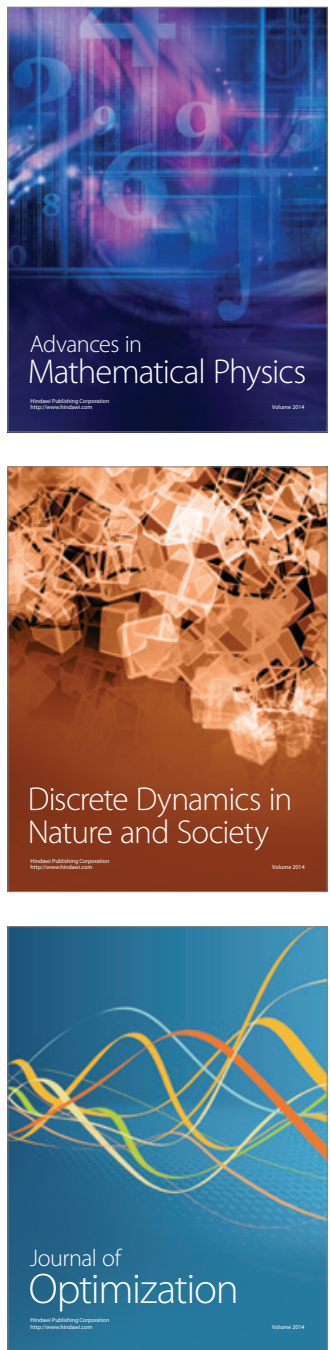